\title{
GLRT Receivers for UWB Systems
}

\author{
Antonio A. D'Amico and Umberto Mengali
}

\begin{abstract}
This letter investigates generalized-likelihood-ratiotest (GLRT) detectors for ultra-wideband (UWB) impulse radio systems employing two alternative signaling schemes. One is the so-called transmitted reference method where, in each time frame, a reference pulse is transmitted prior to the data pulse. The other is the differential transmitted reference scheme, wherein the data pulse received in the previous frame is used as a template waveform in the current frame. The two detectors are compared in terms of bit error rate performance.
\end{abstract}

Index Terms-Generalized likelihood ratio test, transmitted reference schemes, ultra-wideband communications.

\section{INTRODUCTION}

$\mathbf{U}$ LTRA-WIDEBAND (UWB) impulse radio systems convey information by transmitting sequences of subnanosecond pulses. In indoor channels each pulse generates hundreds of echoes that, in principle, might be resolved and combined in a Rake receiver. However the implementation of a Rake is too complex, not only for the large number of fingers needed to capture a significant part of the signal energy, but also because the individual echoes have generally different shapes so that a separate shape estimator should be associated to each finger.

As an alternative to the Rake, a transmitted reference (TR) signaling scheme has been proposed in [1]. The idea is to transmit a reference pulse prior to each data pulse and use the channel response to the former as a template waveform to correlate with the latter. This simple mechanism is extended in [2], [3] where the average of the channel responses to several reference pulses is used as a template waveform. The generalized likelihood ratio test (GLRT) detector for TR systems is investigated in [3], [4] under different signal and channel model assumptions.

The drawback of the TR method is that it wastes power and time resources to transmit the reference pulses. In differential transmitted reference (DTR) systems there are no reference pulses and the received signal over a frame is used as a template waveform for the data pulse in the next frame. The performance of simple DTR receivers is discussed in [3].

The purpose of this letter is twofold. On one side we derive the GLRT detectors for TR and DTR and compare their performance. Note that the detector for TR has already been investigated in [4] and [3]. However in [4] the signal model is different from that adopted here, while in [3] the incoming pulses have all the same shape, which is known at the receiver.

Manuscript received October 28, 2004. The associate editor coordinating the review of this letter and approving it for publication was Dr. Kamran Kiasaleh. This work was supported by the Italian Ministry of Education in the framework of the project PRIMO, FIRB 2001.

The authors are with the Department of Information Engineering, Pisa, Italy (e-mail: \{antonio.damico, umberto.mengali\}@iet.unipi.it).

Digital Object Identifier 10.1109/LCOMM.2005.06032.
Unfortunately the equal shape assumption does not hold with UWB channels where, as opposed to narrowband wireless communications, each path has its own transfer function which depends on the specific reflections, diffractions and scattering incurred in that path [5]. Thus, the individual pulses undergo different distorsions and do not resemble the ideal signal received from a line-of-sight path. For these reasons in the following we take the more realistic view that the pulses have arbitrary and unknown shapes.

The second purpose is to give mathematical foundation to a detection scheme for TR proposed in [3] on heuristic grounds. We show that this scheme is actually the GLRT detector.

The rest of the letter is organized as follows. The GLRT detectors for TR and DTR are derived in the next Section. Performance comparisons are made in Section III and conclusions are drawn in Section IV.

\section{GLRT DETECTORS}

\section{A. Transmitted Reference}

We consider a single-user TR system with binary pulseamplitude modulation (PAM) operating over a multipath channel with additive white Gaussian noise (AWGN). The received signal is

$r(t)=\sum_{i=-\infty}^{\infty} h\left(t-i T_{f}\right)+\sum_{i=-\infty}^{\infty} b_{\left\lfloor i / N_{s}\right\rfloor} h\left(t-i T_{f}-T_{d}\right)+w(t)$

In this formula pulse $h(t)$ is the channel response, whose duration is limited to $0 \leq t \leq T_{m d s}$, where $T_{m d s}$ is the channel's maximum delay spread. Note that $h(t)$ results from a single transmitted pulse and is the aggregate of hundreds of multipath components, each with its own shape. In writing (1) we assume that the channel is static so that $h(t)$ does not change in time. The term $w(t)$ is the AWGN with twosided power spectral density $N_{0} / 2$. The signal component is divided into frames of length $T_{f}$, each containing two pulses at a distance $T_{d}$. The first is the reference, the other conveys information. The data symbols $b_{\left\lfloor i / N_{s}\right\rfloor} \in\{ \pm 1\}$ are independent and identically distributed. The notation $\lfloor\cdot\rfloor$ means "integer part of." From (1) it is seen that the transmission of a single bit lasts $N_{s}$ frames, i.e., the symbol duration is $T_{s}=N_{s} T_{f}$. Note that the pulses in (1) are not time-hopped since we concentrate on a single-user application. Time-hopping might be accommodated but it would make the discussion more involved without affecting the conclusions.

Our objective is to derive the GLRT detector under the following assumptions:

(a) $\quad T_{d}$ is greater than $T_{m d s}$ so that there is no overlap between reference and data pulses. 
(b) The frame period is chosen sufficiently large $\left(T_{f} \geq\right.$ $2 T_{m d s}$ ) to preclude interference between the data pulse in a given frame and the reference pulse in the next frame.

(c) Synchronization is perfect, meaning that the starting times of the frames as well as the first frame in a symbol are known.

To begin we note that the symbols can be detected separately without penalty as they are independent and do not interfere with each other. In particular the decision on $b_{0}$ can be based on the observation of

$$
r(t)=\sum_{i=0}^{N_{s}-1}\left[h\left(t-i T_{f}\right)+b_{0} h\left(t-i T_{f}-T_{d}\right)\right]+w(t)
$$

over the interval $J=\left[0, T_{s}\right)$. If $h(t)$ were known, the minimum bit error rate (BER) would be achieved by application of the maximum likelihood (ML) criterion. This would involve maximizing the metric

$\Gamma\left[r(t) \mid b_{0}, h(t)\right]=2 \int_{J} r(t) s\left[t, b_{0}, h(t)\right] d t-\int_{J} s^{2}\left[t, b_{0}, h(t)\right] d t$

with respect to $b_{0}$, where

$$
s\left[t, b_{0}, h(t)\right]=\sum_{i=0}^{N_{s}-1}\left[h\left(t-i T_{f}\right)+b_{0} h\left(t-i T_{f}-T_{d}\right)\right]
$$

Exploiting the assumptions (a) and (b) and performing some straightforward calculations this would lead to the familiar decision rule

$$
\hat{b}_{0, M L}=\operatorname{sgn}\left[\sum_{i=0}^{N_{s}-1} x_{M L}\left(i T_{f}+T_{d}\right)\right]
$$

where $\operatorname{sgn}[x]= \pm 1$ according to the sign of $x$ and $x_{M L}(t):=$ $r(t) \otimes h(-t)$ is the convolution of $r(t)$ with the matched filter $h(-t)$.

Since $h(t)$ is not known, however, the matched filter cannot be implemented and we must content ourselves with a suboptimal solution. A reasonable approach is the GLRT criterion [6] whereby the maximization of (3) is performed not only over $b_{0}$ but also over all the finite-energy $h(t)$-functions with support $t \in\left[0, T_{m d s}\right)$

$$
\hat{b}_{0, G L R T-T R}=\arg \max _{b_{0}}\left\{\max _{h(t)} \Gamma\left[r(t) \mid b_{0}, h(t)\right]\right\}
$$

The maximization is carried out as follows. Substituting (4) into (3) and bearing in mind assumptions (a) and (b), after some manipulations yields

$$
\begin{aligned}
& \Gamma\left[r(t) \mid b_{0}, h(t)\right]= \\
& 2 \int_{0}^{T_{m d s}}\left\{\left[R_{T R}(t)+b_{0} R_{T R}\left(t+T_{d}\right)\right] h(t)-N_{s} h^{2}(t)\right\} d t
\end{aligned}
$$

with

$$
R_{T R}(t):=\sum_{i=0}^{N_{s}-1} r\left(t+i T_{f}\right)
$$

Keeping $b_{0}$ fixed and using variational techniques [7] produces

$$
\begin{aligned}
& \max _{h(t)} \Gamma\left[r(t) \mid b_{0}, h(t)\right]= \\
& \frac{1}{2 N_{s}} \int_{0}^{T_{m d s}}\left[R_{T R}(t)+b_{0} R_{T R}\left(t+T_{d}\right)\right]^{2} d t
\end{aligned}
$$

Next, expanding the square in the right hand side, discarding terms independent of $b_{0}$, and looking for the new maximum with respect to $b_{0}$ gives the desired result

$$
\hat{b}_{0, G L R T-T R}=\operatorname{sgn}\left[\frac{1}{N_{s}} \int_{0}^{T_{m d s}} R_{T R}(t) R_{T R}\left(t+T_{d}\right) d t\right]
$$

The GLRT-TR detector has the following interpretation. Letting

$$
h_{T R}(t):=\frac{1}{N_{s}} \sum_{i=0}^{N_{s}-1} r\left(t+i T_{f}\right) \quad 0 \leq t<T_{m d s}
$$

and bearing in mind (8), the argument of $\operatorname{sgn}[\cdot]$ in (10) may be rearranged as

$$
\frac{1}{N_{s}} \int_{0}^{T_{m d s}} R_{T R}(t) R_{T R}\left(t+T_{d}\right) d t=\sum_{i=0}^{N_{s}-1} x_{T R}\left(i T_{f}+T_{d}\right)
$$

where $x_{T R}(t):=r(t) \otimes h_{T R}(-t)$. Thus, the GLRT-TR detector is a correlation receiver in which the template waveform is the average of the reference pulses received over one symbol interval. Interesting enough, this scheme coincides with the averaged transmitted reference (ATR) receiver proposed in [3] on heuristic grounds.

\section{B. Differential Transmitted Reference}

In a DTR system the received signal is [3]

$$
r(t)=\sum_{i=-\infty}^{\infty} a_{i} h\left(t-i T_{f}\right)+w(t)
$$

where $a_{i}$ are binary symbols \pm 1 that are related to the data symbols $b_{i}$ by the relation $a_{i}=a_{i-1} b_{\left\lfloor i / N_{s}\right\rfloor}$. All the other parameters are as before. Again, a symbol-by-symbol decision can be made and, in particular, the decision on $b_{0}$ can be based on the observation of

$$
r(t)=\sum_{i=0}^{N_{s}-1} a_{i} h\left(t-i T_{f}\right)+w(t)
$$

over $J=\left[0, T_{s}\right)$.

For $0 \leq i \leq N_{s}-1$ it is easily checked that

$$
a_{i}=\left\{\begin{array}{cc}
b_{0} a_{-1} & \text { for } i \text { even } \\
a_{-1} & \text { for } i \text { odd }
\end{array}\right.
$$

Thus, assuming for simplicity $N_{s}$ even, (14) may be rearranged as

$$
r(t)=\sum_{i=0}^{N_{s} / 2-1}\left[b_{0} h^{\prime}\left(t-i 2 T_{f}\right)+h^{\prime}\left(t-i 2 T_{f}-T_{f}\right)\right]+w(t)
$$

with $h^{\prime}(t)=a_{-1} h(t)$.

Comparing (16) with (2) we note a striking similarity. The signal component in (16) is still divided in frames (of length 
$2 T_{f}$ instead of $T_{f}$ ) and each frame contains two pulses at a distance $T_{f}$ (instead of $T_{d}$ ). The total number of frames is reduced to $N_{s} / 2$. As opposed to (2), the first pulse "conveys information" while the other acts as a "reference". From the point of view of data detection this swapping is inconsequential. Also, function $h^{\prime}(t)$ is no longer the channel response, it is the product $a_{-1} h(t)$. This is irrelevant however as $h(t)$ is unknown and must be estimated. In summary, the detection problem is formally identical to that discussed previously and the solution is easily found with the same reasoning. Letting

$h_{D T R}(t):=\frac{2}{N_{s}} \sum_{i=0}^{N_{s} / 2-1} r\left(t+i 2 T_{f}+T_{f}\right) \quad 0 \leq t<T_{m d s}$

the decision rule turns out to be

$$
\hat{b}_{0, G L R T-D T R}=\operatorname{sgn}\left[\sum_{i=0}^{N_{s} / 2-1} x_{D T R}\left(i 2 T_{f}\right)\right]
$$

with $x_{D T R}(t):=r(t) \otimes h_{D T R}(-t)$. Thus, the GLRT-DTR detector computes the average of the received pulses in the odd frames and correlates it with the received signal in the even frames.

\section{PERFormANCE COMPARISONS}

As mentioned earlier, the GLRT-TR detector coincides with the ATR receiver [3], whose error probability is

$P_{G L R T-T R}=Q\left(\left[\frac{2}{N_{s}}\left(\frac{N_{0}}{2 E_{p}}\right)+\frac{2 W T_{m d s}}{N_{s}^{2}}\left(\frac{N_{0}}{2 E_{p}}\right)^{2}\right]^{-1 / 2}\right)$

where $E_{p}$ is the energy of $h(t)$ and $W$ is the one-sided noise bandwidth of the receiver.

The operation of the GLRT-DTR detector is similar to that of the GLRT-TR, except that the template waveform is computed from the odd frames and the number of "data frames" is $N_{s} / 2$, not $N_{s}$. Thus its error probability may be computed from (19) with the substitution $N_{s} \rightarrow N_{s} / 2$, i.e.,

$$
\begin{aligned}
& P_{G L R T-D T R}= \\
& Q\left(\left[\frac{4}{N_{s}}\left(\frac{N_{0}}{2 E_{p}}\right)+\frac{8 W T_{m d s}}{N_{s}^{2}}\left(\frac{N_{0}}{2 E_{p}}\right)^{2}\right]^{-1 / 2}\right)
\end{aligned}
$$

On the other hand, the energy per symbol in GLRT-TR is $E_{s}=2 N_{s} E_{p}$ while in GLRT-DTR is $E_{s}=N_{s} E_{p}$. Expressing (19) and (20) in terms of $E_{s}$ yields the same result

$$
P_{G L R T}=Q\left(\left[\frac{2 N_{0}}{E_{s}}+2 W T_{m d s}\left(\frac{N_{0}}{E_{s}}\right)^{2}\right]^{-1 / 2}\right)
$$

which shows that the two detectors have the same energy efficiency.

\section{Conclusions}

The GLRT detectors for UWB-TR and UWB-DTR systems are correlation receivers in which the template waveform is computed as the average of the channel responses to some "reference" pulses. In the TR case the average is taken over the true reference pulses and the correlation is computed exploiting all the frames in a symbol. In the DTR case the average is taken over the odd frames and the correlation is computed exploiting the even frames. It has been shown that the two receivers have the same energy efficiency.

\section{REFERENCES}

[1] R. Hoctor and H. Tomlinson, "Delay-hopped transmitted-reference RF communications," in Proc. IEEE UWBST, 2002, pp. 265-269.

[2] J. D. Choi and W. E. Stark, "Performance of ultra-wideband communications with suboptimal receivers in multipath channels," IEEE J. Select. Areas Commun., vol. 20, pp. 1754-1766, Dec. 2002.

[3] Y.-L. Chao and R. A. Scholtz, "Optimal and suboptimal receivers for ultra-wideband transmitted reference systems," in Proc. GLOBECOM'03, vol. 2, pp. 759-763, Dec. 2003.

[4] S. Franz and U. Mitra, "On optimal data detection for UWB transmitted reference systems," in Proc. GLOBECOM'03, vol. 2, pp. 744-748, Dec. 2003.

[5] R. C. Qiu, "A study of the ultra-wideband wireless propagation channel and optimum UWB receiver design," IEEE J. Select. Areas Commun., vol 20, pp. 1628-1637, Dec. 2002.

[6] S. M. Kay, Fundamentals of Statistical Signal Processing: Detection Theory. Upper Saddle River, NJ: Prentice Hall, Inc., 1998.

[7] D. A. Pierre, Optimization Theory with Applications. New York: Dover Publications, Inc., 1986. 